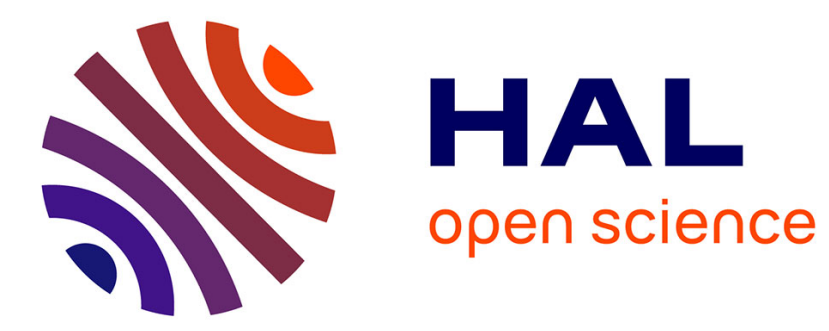

\title{
A phenomenological approach to phase transition in classical field theory
}

Francesco Dell'Isola, A. Romano

\section{To cite this version:}

Francesco Dell'Isola, A. Romano. A phenomenological approach to phase transition in classical field theory. International Journal of Engineering Science, 1987, pp.7. hal-00502977

\section{HAL Id: hal-00502977 \\ https://hal.science/hal-00502977}

Submitted on 16 Jul 2010

HAL is a multi-disciplinary open access archive for the deposit and dissemination of scientific research documents, whether they are published or not. The documents may come from teaching and research institutions in France or abroad, or from public or private research centers.
L'archive ouverte pluridisciplinaire HAL, est destinée au dépôt et à la diffusion de documents scientifiques de niveau recherche, publiés ou non, émanant des établissements d'enseignement et de recherche français ou étrangers, des laboratoires publics ou privés. 


\title{
A PHENOMENOLOGICAL APPROACH TO PHASE TRANSITION IN CLASSICAL FIELD THEORYं
}

\author{
F. DELL'ISOLA and A. ROMANO \\ Dipartimento di Matematica e sue Applicazioni dell'Universita' di Napoli, Via Mezzocannone, \\ 8-80134, Napoli, Italy \\ (Communicated by E. S. SUHUBI)
}

\begin{abstract}
In this paper we propose some surface constitutive equations when added to the balance laws and jump conditions allow us to formulate in principle a boundary value problem for phase transitions modelled as a continuum with an interface.
\end{abstract}

\section{INTRODUCTION}

In this paper we regard a physical system where phase transition is taking place in a continuum which is composed of two tridimensional continua $C_{1}$ and $C_{2}$ occupying contiguous regions shared by a surface $S$.

Moreover, in order to describe "surface" phenomena occurring at the interface sharing the phases we regard surface $S$ as a bidimensional continuum carrying thermomechanical properties.

Finally, we assume for the continuous system $\left(C_{i}, S\right)$ the general thermomechanical balance laws (GTBL) derived in [1] for continua with non material and adsorbing interface.

The necessity of employing (GBTL) can be understood if we note that, in growth and reduction processes occurring during phase transition, the total amount of particles constituting $S$ varies, because of at least two reasons:

(i) separation regions between bulk media are created or destroyed.

(ii) different thermomechanical conditions can affect the surface density.

This implies that, if a mass is to be assigned to $S$, it can vary, so that $S$ has to be adsorbing. Moreover, the particles forming the interface $S$ are continually changing during the process, so that it becomes impossible to use material coordinates in order to describe the interface itself. Therefore, surface parameters, (time dependent or not), describing $S$ have just a geometrical meaning. For the same reason, we cannot choose a reference configuration for $S$, so that it can be described in the Eulerian formalism only (see [2]).

It is obvious that together with GTBL some constitutive equations have to be specified, in order to model the properties of both the bulk materials constituting the phases and the interface sharing them.

Concerning this last point we first of all underline that in this paper we will always assume to deal with "viscous" materials.

While the meaning of this statement is clear when referred to tridimensional continua, we need to be more precise when we refer to bidimensional ones.

To this aim we introduce the surface velocity field $\mathbf{v}$ which allows us to represent surface linear momentum in the usual form $\rho_{\sigma} \mathbf{v}$, with $\rho_{\sigma}$ surface mass density. Moreover we can assume that its tangential component $\mathbf{v}_{\tau}$ coincides with the tangential field specifying the velocity with which surface quantities are transported along $S$ (all the considerations concerning physical meaning of $\mathbf{v}$ and its relation with $\mathbf{v}_{\tau}$ and GTBL can be found in [1], see also [3]).

We can now observe that the viscosity of the interface $S$ has to be expressed not only with usual assumptions about surface stress tensor but also with the following hypothesis about the aforesaid surface velocity field:

(a) The tangential components of velocity on both sides of $S$ are equal to the tangential velocity $\mathbf{v}_{\tau}$. This means that at any instant the particles of both phases

† Work supported by G.N.F.M. of the Italian C.N.R. 
adhere to the interface. Another hypothesis we will assume, as it in fact characterizes phase transition phenomena:

(b) Constitutive equations of both continua $C_{i}$ do not change because of matter transport through $S$. This means that matter completely changes its original properties when entering the arrival region.

We finally observe that the assumption $v_{n}=c_{n}\left(c_{n}\right.$ being geometric normal speed of the surface $S$ ), cannot at all be justified, when our model is intended to describe phase transition.

This is obvious when we recall physical interpretation of the surface field $v_{n}$ given in [1], which lead us to identify this velocity as that with which material particle "instantaneously" laying on the interface is moving. $\uparrow$

Moreover it will result that the difference $v_{n}-c_{n}$ depends on the modalities with which phase transition is taking place, as it is determined by the surface evolution equations we are going to obtain.

We will assume that entropy principle imposes restrictions on constitutive equations for both bulk materials and interface.

This means that we cannot specify for instance the modalities of mass flux, dissipation, linear momentum and energy exchange through the interface in a completely arbitrary way as every set of constitutive equations we propose for describing our systems has to satisfy the residual entropy inequality.

Uniqueness theorem for the solution of Moving Boundary problem relative to compressible fluids (Graff [5], Serrin [6]) together with the structure of entropy inequality suggested us to introduce six new surface constitutive equations. $\ddagger$

The first one specifies the flux of mass through the surface, once temperature and other independent quantities are given; the second one represents a generalization of the well-known relation existing at the equilibrium between Gibbs' potentials; the third one gives a link between $v_{n}-c_{n}$ and the normal tension exerted on the interface, which, at equilibrium, reduces to the well-known Laplace's formula; the fourth one establishes a generalization of Newton's law for heat conduction (when a jump of temperature exists across the interface); the fifth one becomes Clausius-Duhem inequality for the entropy production, when the jump of temperature vanishes; finally the sixth equation is a physically reasonable relation between the amount of mass leaving at the same time both phases.

In conclusion, we observe that in general it is necessary to introduce some constitutive equations connecting surface and bulk properties if we want to describe, by means of the constructed mathematical model, phenomena occurring when interfaces are made up with the same materials which they are sharing, just as it happens in phase transition.

Of course, some simplifications can be introduced in our model.

For example, if it is possible to neglect the mass transfer phenomena on the interface, it has to be assumed that surface tension depends only on temperature.

Finally, if it is possible to neglect every surfacial phenomenon, $S$ will become a simple discontinuity surface.

\section{LOCAL BALANCE EQUATIONS AND PRELIMINARY CONSIDERATIONS ABOUT ENTROPY INEQUALITY}

For sake of completeness we quote here GTBL we derived in [1]:

$$
\begin{aligned}
& \frac{\partial \rho}{\partial t}+\operatorname{div} \rho \dot{\mathbf{x}}=0 ; \quad \rho \ddot{\mathbf{x}}=\operatorname{div} \mathbf{T}+\rho \mathbf{b} ; \quad \mathbf{T}=\mathbf{T}^{T} \\
& \rho \dot{\varepsilon}=\mathbf{T}: \operatorname{grad} \dot{\mathbf{x}}-\operatorname{div} \mathbf{h}+\rho r ; \quad \rho \dot{\eta} \geq-\operatorname{div}(\mathbf{h} / \theta)+\rho r / \theta
\end{aligned}
$$

$\dagger$ A careful description of experimental evidence available about physical properties of the interfaces between different phases can be found in [4].

$¥$ It seems possible that the restrictions on constitutive equations determined by entropy principle could be found applying the general method of Lagrange multipliers proposed by I-Shih Liu [7] for tridimensional continua. 


$$
\left.\begin{array}{c}
\frac{\delta_{n}}{\delta t} \rho_{\sigma}-2 H c_{n} \rho_{\sigma}+\operatorname{div}_{S} \rho_{\sigma} \mathbf{v}_{\tau}+\llbracket \rho(\dot{\mathbf{x}}-\mathbf{c}) \rrbracket \cdot \mathbf{n}=0 \\
\rho_{\sigma} \frac{\delta_{n}}{\delta t} \mathbf{v}+\rho_{\sigma} \nabla_{S} \mathbf{v} \cdot \mathbf{v}_{\tau}-\operatorname{div}_{S} \mathbf{T}_{\sigma}+\llbracket \rho(\dot{\mathbf{x}}-\mathbf{v}) \otimes(\dot{\mathbf{x}}-\mathbf{c})-\mathbf{T} \rrbracket \cdot \mathbf{n}=0 . \\
\rho_{\sigma} \frac{\delta_{n}}{\delta t} \varepsilon_{\sigma}+\rho_{\sigma} \nabla_{S} \varepsilon_{\sigma} \cdot \mathbf{v}_{\tau}-\mathbf{T}_{\sigma}: \nabla_{S} \mathbf{v}+\operatorname{div}_{S} \mathbf{h}_{\sigma} \\
\left.+\llbracket \rho\left(\frac{1}{2}(\dot{\mathbf{x}}-\mathbf{v})^{2}+\left(\varepsilon-\varepsilon_{\sigma}\right)\right)(\dot{\mathbf{x}}-\mathbf{c})-(\dot{\mathbf{x}}-\mathbf{v}) \cdot \mathbf{T}+\mathbf{h}\right] \cdot \mathbf{n}=0
\end{array}\right\}
$$

where: $\rho$ is volume mass density, $\rho_{\sigma}$ is surface mass density, $\dot{\mathbf{x}}$ is the velocity field in tridimensional continua $C_{i}, \mathbf{v}$ is the surface velocity field, $\mathbf{T}$ is the Cauchy stress tensor in $C_{i}, \mathbf{T}_{\sigma}$ is the surface stress tensor, whose tangential components are $T_{\sigma}^{\alpha \beta}, \rho \mathbf{b}$ and $\rho_{\sigma} \mathbf{b}_{\sigma}$ are given volume and surface force density, $\varepsilon$ is mass internal energy density in $C_{i}, \varepsilon_{\sigma}$ is surface mass internal energy density, $\mathbf{h}$ and $\mathbf{h}_{\sigma}$ are volume and surface heat fluxes, $\eta$ and $\eta_{\sigma}$ are volume and surface mass entropy densities, $r$ and $r_{\sigma}$ are mass heat production density in $C_{i}$ and on $S, \theta$ and $\theta_{\sigma}$ are volume and surface temperature, $\psi \equiv \varepsilon-\theta \eta$ and, in the same way, $\psi_{\sigma}=\varepsilon_{\sigma}-\theta_{\sigma} \eta_{\sigma}, H$ is the mean curvature of the surface $S$, whose normal is $\mathbf{n}$ and normal velocity is $c_{n}$. The symbols $\delta_{n} / \delta t, \nabla_{S}$, $\operatorname{div}_{S}$ respectively denote Thomas' derivative, surface gradient and surface divergence, while, for the generic function $f_{\sigma}$, is:

$$
f_{\sigma}^{\prime} \equiv \frac{\delta_{n}}{\delta t} f_{\sigma}+\nabla_{S} f_{\sigma} \cdot \mathbf{v}_{\tau}
$$

In the following we will not assume the continuity of $\theta$ across the interface. On the other hand for sake of simplicity, we will suppose that all surface quantities depend only on $\rho_{\sigma}, \theta_{\sigma}, \nabla_{S} \theta_{\sigma}, \nabla_{S} \mathbf{v}$. Moreover we will assume, coherently with the hypothesis of continuity for tangential velocity, that: the interface is a viscous membrane, i.e. $\mathbf{T}_{\sigma}=\boldsymbol{\gamma} \mathbf{I}_{\sigma}+\tilde{\mathbf{T}}_{\sigma}$. Here $\tilde{\mathbf{T}}_{\sigma}$ is the dynamical part of $\mathbf{T}_{\sigma}$, whose components in the coordinates $u^{\alpha}$ are: $\tilde{T}_{\sigma \gamma}^{\alpha}=\lambda \sigma_{\gamma}^{\nu} a_{\gamma}^{\alpha}+\mu \sigma_{\gamma}^{\alpha}$, being $\sigma_{\gamma}^{\alpha} \equiv 2 v_{\tau \mid \gamma}^{\alpha}-b_{\gamma}^{\alpha} v_{n}$, and $a_{\gamma}^{\alpha}, b_{\gamma}^{\alpha}$ the coefficients of the first and second fundamental quadratic forms on $S$ (see [8]).

Finally, we will suppose that the following relations which can be deduced from the entropy principle in the case of a real material bidimensional continuum, still hold:

$$
\left.\begin{array}{l}
\psi_{\sigma}=\psi_{\sigma}\left(\rho_{\sigma}, \dot{\theta}_{\sigma}\right) \quad \eta_{\sigma}=-\frac{\partial \psi_{\sigma}}{\partial \theta} \quad \gamma=-\rho_{\sigma}^{2} \frac{\partial \psi_{\sigma}}{\partial \rho_{\sigma}} \\
\mathbf{h}_{\sigma}=-k\left(\rho_{\sigma}, \theta_{\sigma}\right) \nabla_{s} \theta_{\sigma} \quad \text { with } k>0
\end{array}\right\}
$$

We now rewrite the entropy inequality $(2.5)$ in the form:

$$
\left.\begin{array}{l}
-\rho_{\sigma}\left(\psi_{\sigma}^{\prime}+\eta_{\sigma} \theta_{\sigma}^{\prime}\right)+\mathbf{T}_{\sigma}: \nabla_{S} \mathbf{v}-\frac{\mathbf{h}_{\sigma}}{\theta_{\sigma}} \cdot \nabla_{S} \theta_{\sigma}+\psi_{\sigma} \llbracket J \rrbracket+ \\
-\llbracket J\left(\frac{1}{2}(\dot{\mathbf{x}}-\mathbf{v})^{2}+\psi\right)-(\dot{\mathbf{x}}-\mathbf{v}) \cdot \mathbf{T} \cdot \mathbf{n} \rrbracket+\llbracket\left(\theta_{\sigma} / \theta-1\right) \mathbf{h} \rrbracket \cdot \mathbf{n} \geq 0
\end{array}\right\}
$$

whẹre $J^{ \pm} \equiv \rho^{ \pm}(\dot{\mathbf{x}}-\mathbf{c})^{ \pm} \cdot \mathbf{n}$. 
As anticipated in Section 1 we will limit ourselves to choose particular classes of constitutive relations for the interface which only assure that suitable groups of terms in (2.8) are separately positive.

Indeed let us consider the first term appearing in (2.8). Because of assumption (2.7) it reads:

$$
\rho_{\sigma}\left(\frac{\partial \psi_{\sigma}}{\partial \theta_{\sigma}} \theta_{\sigma}^{\prime}+\frac{\partial \psi_{\sigma}}{\partial \rho_{\sigma}} \rho_{\sigma}^{\prime}+\eta_{\sigma} \theta_{\sigma}^{\prime}\right) .
$$

The coordinate explicit form of the second term in formula (2.8) is:

$$
\mathbf{T}_{\alpha}: \nabla_{S} \mathbf{v}=\gamma \sigma_{\alpha}^{\alpha}+\tilde{T}_{\sigma \gamma}^{\alpha} \alpha_{\alpha}^{\gamma}
$$

Moreover balance eqn (2.2) for $\rho_{\sigma}$ implies that:

$$
\rho_{o}^{\prime}=-\rho_{\sigma} \sigma_{\alpha}^{\alpha}-\llbracket J \rrbracket+2 H\left(c_{n}-v_{n}\right) \rho_{\sigma}
$$

Substituting in (2.10) this last relation and adding to the so obtained expression the second member of equality (2.10) we can collect all the terms containing $\theta_{\sigma}^{\prime}$ and $\sigma_{\alpha}^{\alpha}$. The possibility we have to arbitrarily choose them leads us to impose that the expressions multiplying them vanish. In this way we have found another justification of assumptions $(2.7)_{2,3}$.

Moreover it is natural to assume that the dissipative terms:

$$
\mathbf{h}_{\sigma} / \theta_{\sigma} \cdot \nabla_{s} \theta_{\sigma} \quad \bar{T}_{\sigma \gamma}^{\alpha} \sigma_{\alpha}^{\gamma}
$$

are both always positive. This is implied by $(2.7)_{4}$ and (see for instance [8]) by relations:

$$
\lambda \geqslant 0, \quad \mu \geqslant 0 .
$$

\section{FURTHER CONSIDERATIONS ABOUT THE REDUCED ENTROPY INEQUALITY}

Under the assumptions quoted in the previous section, it results that among the quantities appearing in formula (2.9) we have still to take into account only the following one:

$$
\rho_{\sigma} \frac{\partial \psi_{\sigma}}{\partial \rho_{\sigma}}\left\{2 H\left(c_{n}-v_{n}\right) \rho_{\sigma}-\llbracket I \rrbracket\right\}
$$

which has to be added to the remaining terms of (2.8).

We can therefore conclude that the reduced entropy inequality will be verified if the following one holds:

$$
\begin{aligned}
\llbracket \rho\left(\frac{1}{2}(\dot{\mathbf{x}}-\mathbf{v})^{2}+g-g_{\sigma}\right)\left(\dot{x}_{n}-c_{n}\right) \rrbracket+\left(v_{n}-c_{n}\right)(\llbracket \tilde{p} \rrbracket-2 \dot{H} \gamma) \\
+\llbracket\left(\theta-\theta_{\sigma}\right)\left\{\rho \eta(\dot{\mathbf{x}}-\mathbf{c})-\frac{\mathbf{h}}{\theta}\right\} \rrbracket \cdot \mathbf{n} \geqslant 0,
\end{aligned}
$$

where $\bar{p} \equiv-\mathbf{n} \cdot \mathbf{T} \cdot \mathbf{n}, g \equiv \psi+\tilde{p} / \rho$, and $g_{\sigma} \equiv \psi_{\sigma}-\gamma / \rho_{\sigma}$.

We note that $g^{ \pm}$coincides, in the case of a perfect fluid, with the limit at $S$ of Gibbs' potential, while $g_{\sigma}$ can be regarded as surface density of the same potential.

Repeatedly applying in (3.2) the following obvious equality:

$$
\llbracket A B \rrbracket=\frac{1}{2}\left(A^{+}+A^{-}\right) \llbracket B \rrbracket+\frac{1}{2}\left(B^{+}+B^{-}\right) \llbracket A \rrbracket
$$

we can see that, if the following set of relations holds, we are assured that inequality 
(3.2) is verified:

$$
\begin{gathered}
\left\{\left(\frac{1}{2}(\dot{\mathbf{x}}-\mathbf{v})^{2}+g\right)^{+}+\left(\frac{1}{2}(\dot{\mathbf{x}}-\mathbf{v})^{2}+g\right)^{-}-2 g_{\sigma}\right\}=-\alpha_{1} \llbracket J \rrbracket, \\
\llbracket \frac{1}{2}(\dot{\mathbf{x}}-\mathbf{v})^{2}+g \rrbracket=-\alpha_{2}\left(J^{+}+J^{-}\right), \\
\quad\{2 H \gamma-\llbracket \bar{p} \rrbracket\}=-\alpha_{3}\left(v_{n}-c_{n}\right), \\
\llbracket \theta \rrbracket=-\alpha_{4}\left\{\frac{\mathbf{h}^{+} \cdot \mathbf{n}}{\theta^{+}}+\frac{\mathbf{h}^{-} \cdot \mathbf{n}}{\theta^{-}}+J^{+} \eta^{+}+J^{-} \eta^{-}\right\}, \\
-\alpha_{5} \llbracket \frac{h}{\theta} \cdot \mathbf{n}-J \eta \rrbracket=\left\{\left(\theta_{\sigma}-\theta\right)^{+}+\left(\theta_{\sigma}-\theta\right)^{-}\right\},
\end{gathered}
$$

where $\alpha_{i}$ are suitable positive functions of all surface relevant quantities.

\section{CONCLUDING REMARKS}

In order to describe surface phenomena occurring because of the presence of an interface between two phases we introduced the following set of surface fields (S1): $\rho_{\sigma}$ (surface mass density), $\rho^{ \pm}$(limits at the interface of bulk mass density fields), $\theta^{ \pm}$ (limits at the interface of bulk temperature fields), $\theta_{\sigma}$ (surface temperature field), $\mathbf{v}$ (surface velocity field with which $\rho_{\sigma}$ travels), $c_{n}$ (geometrical normal velocity of the interface), $\dot{\mathbf{x}}^{ \pm}$(limits at the interface of velocity bulk field) together with all those listed at the end of Section 1, quantities which already were assumed to be determined by those belonging to $\mathrm{S} 1$.

It is now clear that the sixteen quantities (S1) do not represent an "independent" set of surface quantities. In fact thermomechanical balance laws determine five scalar surface evolution equations (set of jump eqns $(2.2),(2.3),(2.4)$ so that we are led to choose as a candidate set of independent $\uparrow$ surface quantities the following one:

$$
c_{n}, v_{n}, \rho_{o}, \mathbf{v}_{\tau} .
$$

We conclude that a certain number of "constitutive" relations among the fields in the set (S1) have to be postulated in order to make our model complete. In this connection we remark that eqns (3.3)-(3.7), together with hypothesis (a):

$$
\dot{\mathbf{x}}_{\tau}^{+}=\dot{\mathbf{x}}_{\tau}^{-}=\mathbf{v}_{\tau}
$$

(which can be regarded as a particular constitutive equation), represent nine of these relations.

It could seem now that we lack for two other relations. This is not exactly the case as it will be evident from the following considerations:

Let us assume $a b$ absurdo that two other relations among the quantities belonging to (S1) need to be postulated beyond (3.3)-(3.7), (4.1).

We should have in this case the possibility to express ail the quantities in the set (S1) in terms of those appearing in (S2) so that in both regions $C_{i}(i=1,2)$ a Moving-Boundary Problem (MBP) for the set of eqns (2.1) could be posed in which on the common (to $C_{1}$ and $C_{2}$ ) boundary $S$ the values of the quantities:

$$
\rho^{ \pm}, \theta^{ \pm}, U^{ \pm} \equiv(\dot{\mathbf{x}}-\mathbf{c})^{ \pm} \cdot \mathbf{n}
$$

are given (n is the outward-pointing normal vector to the surface $S$ ).

Now it is well-known (see Uniqueness Theorems shown in $[5,6]$ ) that boundary conditions of this MBP are not redundant only when $\rho^{ \pm}$are given only in those points of $S$ where $U^{+}$is less than zero and $U^{-}$is greater than zero.

$\dagger$ This means that we single out a set of quantities which are sufficient to determine the state of the interface so that in dependence on them it is possible to express all those quantities occurring in the balance laws. 
On the other hand, in phase transition, it is reasonable to assume that $J^{ \pm} \equiv \rho^{ \pm} U^{ \pm}$ have the same sign, so that the quoted MBP is surely not well-posed. In conclusion it results that just one more (suitable) constitutive relation has to be added to (3.3)-(3.7) in order to formulate a Free-Moving-Boundary Problem (FMBP) for eqns (2.1) which could have reasonable chances to be well-posed. $\dagger$

We postulate that this constitutive equation is given in the case of phase transition by the relations:

$$
J^{+}=\alpha_{6} J^{-}
$$

where $\alpha_{6}$ is a positive function of all S1 variables.

From a mathematical point of view the addition of formula (4.2) to the set of eqns (3.3)-(3.7), (4.1) allows us to specify all the quantities in S1 necessary to assure Serrin's Uniqueness Theorem is true when all quantities in S2 are given, so that the evolution eqns (2.2)-(2.6) can be regarded as equations in which the only unknowns are exactly $\mathrm{S} 2$ quantities.

From a physical point of view formula (4.2) express the circumstance that during phase transition the interface cannot grow because of mass transport from both phases.

We conclude this number underlining that we propose to describe all properties of a particular material which can present itself in two or more phases assigning bulk constitutive equations for every of these phases together with all functions $\alpha_{i}$ $(i=1, \ldots, 5)$ pertinent to every possible kind of interface. It is logically possible to construct a more general theory, in which properties of materials are modelled with more complex types of constitutive equations. Only a close inquiry of the wealth of experimental evidence available about phase transition can allow us to ascertain if this greater generality is really necessary. At equilibrium, the set of balance equations and
of relations (3.3)-(3.7), (4.1), (4.2) reduces to:

$$
\left.\begin{array}{c}
\theta^{+}=\theta^{-}=\theta_{\sigma}=\theta_{1}=\theta_{2}=\text { const. } ; \quad \dot{\mathbf{x}}_{1}=\dot{\mathbf{x}}_{2}=\mathbf{v}_{\tau}=\mathbf{0} ; \\
v_{n}=c_{n}=0 ; \quad \operatorname{div}_{S} \mathbf{T}_{\sigma}=-\llbracket \mathbf{T} \cdot \mathbf{n} \rrbracket ; \\
\llbracket g \rrbracket=0 ; \quad g^{+}+g^{-}=2 g_{\sigma} ; \\
2 H \gamma-\llbracket \tilde{p} \rrbracket=0 ;
\end{array}\right\}
$$

Equations (4.3) result to be exactly those classically assumed hold at equilibrium. Moreover, although eqn (3.5) is in general independent of all other eqns (3.3)-(3.7) and evolution eqns $(2.1)-(2.6)$, it has to be remarked that at equilibrium $(4.3)$, stemming from (3.5) is implied by (4.3) $)_{4}$ stemming from (2.3).

We conclude observing that:

(i) Formulas (3.3), (3.4) link the mass respectively adsorbed by and crossing the interface to surface and dynamical free energy;

(ii) Formula (3.5) expresses the physically meaningful circumstance that the phase transition speed is driven by the pressure derangement across the interface;

(iii) Formula (3.6) reduces, when the thermomechanical phenomenon under consideration is purely thermical and stationary (i.e. all the velocity fields vanish, while phase transition is not taking place, so that it results $h_{n}^{+}=h_{n}^{-}$), to the well-known Newton's law for heat conduction when temperature jumps are present.

(iv) Formula (3.7) is a surface form of Clausius-Duhem law for the entropy
production.

(v) It could seem that, when:

$$
\theta_{\sigma}=\theta^{+}=\theta^{-}
$$
(i.e. when thermal resistivity of the interface can be neglected) formulas (3.6), (3.7)
may imply that:

$$
\llbracket \frac{\mathbf{h}}{\theta} \cdot \mathbf{n}+J \eta \rrbracket=\frac{\mathbf{h}^{+} \cdot \mathbf{n}}{\theta^{+}}+\frac{\mathbf{h}^{-} \cdot \mathbf{n}}{\theta^{-}}+J^{+} \eta^{+}+J^{-} \eta^{-}=0 .
$$


We underline that this is not the case: in fact it has to be observed that assuming equalities (4.4) true along every evolution of considered system actually means to assume that coefficients $\alpha_{4}$ and $\alpha_{5}$ are both zero for the quoted system.

\section{REFERENCES}

[1] F. DELL'ISOLA and A. ROMANO, On the derivation of thermomechanical balance equations for continuous systems with a non material interface. Int. J. Engng Sci. 25, 1459-1468 (1987).

[2] F. DELL'ISOLA and A. ROMANO, On a general balance law for continua with an interface. (to appear in Ricerche di Matematica Napoli).

[3] W. KOSINSKI, Field Singularities and Wave Analysis in Continuum Mechanics. PWN-Polish Scientific Publisher Warsaw (1987).

[4] J. N. MURREL and E. A. BOUCHER, Properties of Liquids and Solutions. Wiley, Chichester (1982).

[5] D. GRAFFI, Il teorema di unicita della dinamica dei fluidi compressibili. J. Ratl Mech. Anal. 2, 99-106 (1953).

[6] J. SERRIN, On the uniqueness of compressible fuid motions. Arch. Ratl Mech. Anal. 34, 271-288 (1959).

[7] I. S. LIU "Method of Lagrangian Multipliers for exploitation of the entropy principle." Arch. Rat. Mech. Anal., vol. 46, 1972.

[8] K. A. LINDSAY and B. STRAUGHAN "A thermodynamical viscous interface theory and associated stability problems." Arch. Rat. Mech. Anal., vol. 71, 4, pp. 307-326, 1979.

(Received 5 February 1987) 